\title{
ESTILOS DE LIDERAZGO EN INSTITUCIONES DE EDUCACIÓN MEDIA
}

\section{LEADERSHIP STYLES IN MEDIA EDUCATION INSTITUTIONS}

Autor 1. Sandra Migdonia Tovar Trujillo

Perfil: Licenciada Administración, Educativa Magister en Negocios

Internacionales y en Administración de las organizaciones, integrante del grupo de investigación Cananguchales Escuela de Ciencias Administrativas, Contables, Económicas y de Negocios de la Universidad Nacional abierta a Distancia UNAD.

ORCID: https://orcid.org/0000-0002-5325-2725

Correo electrónico: sandra.tovar@unad.edu.co

\section{Autor 2. Shyrley Rocio Vargas Paredes}

Perfil: Administradora de empresas, magister en administración de las organizaciones, líder del grupo de investigación Cananguchales Escuela de Ciencias Administrativas, Contables, Económicas y de Negocios de la Universidad Nacional abierta a Distancia UNAD.

ORCID: https://orcid.org/0000-0002-6224-9341

Correo electrónico: Shyrley.paredes@unad.edu.co

Autor 3. Manuel Ignacio Martínez Arce

Perfil: Estudiante del programa Administración de Empresas de la Escuela de Ciencias Administrativas, Contables, Económicas y de Negocios de la Universidad Nacional abierta a Distancia UNAD.

Correo electrónico: manmar777@hotmail.com

\section{RESUMEN}

El presente documento de trabajo expone la investigación en curso que aborda los estilos de liderazgo desde el modelo de los 4 marcos en instituciones de educación media de la ciudad de Neiva. El objetivo determinar los estilos de liderazgo en instituciones de educación media: una mirada desde el modelo de los 4 marcos, aplicado a los rectores de las instituciones en Neiva.

La investigación se desarrolla mediante el enfoque cuantitativo, de tipo exploratorio y descriptivo que permitirá identificar los estilos de liderazgos que tienen los rectores de las instituciones de educación media en la Ciudad de Neiva. El instrumento mide 4: conductas, estilos de liderazgo, estimación global e información, cada una con un número significativo de ítem para seleccionar, lo anterior, hace evaluación de los estilos de liderazgo como:

simbólico, político, estructural y de recursos humanos.

Palabras clave: Liderazgo, educación, instituciones educativas 

educativo, sino en lo económico, el recurso humano y el desarrollo organizacional, además el impacto social y la trazabilidad de los procesos.

Consecuente con lo anterior (Ministeria de Educación Nacional, 2007), la visión y valores claros ayudan a que el liderazgo sea distribuido y a tomar decisiones en el colegio. Si todos conocen los valores del colegio, así como la dirección hacia donde van, las decisiones se pueden tomar de acuerdo con ese contexto, lo que permite repartir el liderazgo.

De esta manera, repartir el liderazgo, permite potenciar el comportamiento humano en las instituciones educativas, donde todos conformar el recurso humano y son esenciales para lograr los objetivos y promover el desarrollo organizacional. Para las instituciones educativas, hoy es importante la marca empresarial para esto se debe partir por fortalecer la marca personal de cada uno de los miembros del equipo, es así que una institución educativa con marca empresarial tiene características diferenciadoras para contribuir al tejido social

Son muchos quienes se atreven a hablar de liderazgo, pero pocos los que realmente lo llevan a la práctica, ya que es una temática bastante amplia que requiere un estudio minucioso, por ello Bennis afirma que:

De entre todas las áreas oscuras y confusas en psicología social, la del liderazgo lucha indudablemente por alcanzar el primer puesto. [...] probablemente sea el Liderazgo más que cualquier otro tópico en ciencias sociales, el tema del que más se ha escrito, pero sobre el que menos se conoce. (Bennis, 1989).

Y aún sigue siendo una idea muy válida en estos días, teniendo en cuenta que el liderazgo al igual que muchas cosas de nuestro alrededor evolucionan de acuerdo con las necesidades actuales del entorno, puesto que "el liderazgo es siempre dependiente del contexto, pero el contexto es establecido por las relaciones" (Wheatley, 1994) que se establecen en los diferentes campos donde se da aplicabilidad a dicho concepto.

De una forma simple Sánchez nos afirma que el Liderazgo "es la capacidad de hacer que otros te sigan con el convencimiento que tus ideas permitirán el avance de la organización y sus miembros" (2012. p. 8), siendo entonces un conjunto de habilidades gerenciales y cualidades directivas para actuar en un grupo de personas en la búsqueda y el alcance de objetivos comunes que benefician a todas las partes, teniendo una influencia positiva que conlleve a la realización de tareas que tienen un fin específico.

Ser un líder implica una gran variedad de virtudes que permitan guiar su grupo de trabajo, la persona debe ser un visionario, con capacidad de comunicación, de establecer ideas y saberlas llevar a cabo, de conducir y reconducir cada uno de los pasos que se desarrollan dentro de una agrupación de personas que "centra sus esfuerzos en alcanzar los objetivos establecidos en la organización" (Sánchez M., 2009).

Ser líder se requiere de capacidades que generen cambios positivos y de esta forma encaminar todos los recursos organizacionales de manera efectiva. En la observación a las instituciones se demuestra que no hay claridad en el papel de los administradores y/o gerentes de las instituciones educativas para el fortalecimiento del desarrollo organizacional a través de su liderazgo. Planteado de esta forma se busca analizar a los establecimientos educativos desde lo organizacional para conocer sobre el desarrollo de la función del rector, 




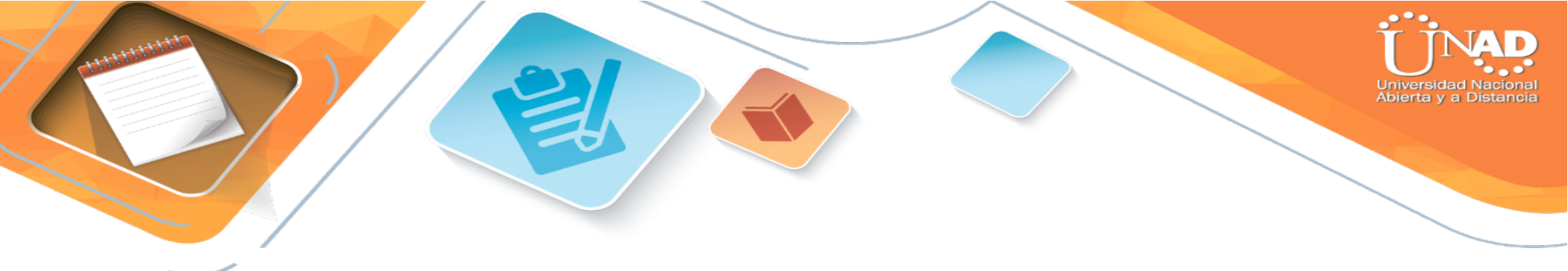

teórico, plantear hipótesis, así mismo, mediante el diseño de la investigación, aplicar las técnicas e instrumentos propuestos para la recolección de datos y medición por medio de procedimientos estandarizados.

La investigación es de tipo descriptivo y de corte transversal, donde los datos se recolectaron en un solo momento y se pretende describir el conocimiento y el manejo de la parte financiera de las mujeres rules de la ciudad de Neiva. Se centra en recolectar la información del problema. El componente descriptivo se desarrolla a través de la medición de cada una de las variables de estudio.

Población Universo: Instituciones de Educación Media de la ciudad de Neiva, departamento del Huila.

Muestra: Rectores y Coordinadores académicos de las Instituciones de Educación Media de la ciudad de Neiva

Tipo de muestreo: el muestreo será no probabilístico (Banerjeey \& Chaudhury, 2010). En esta investigación, los participantes se agrupan por sexo (hombres/mujeres) y cargo (Rectores). Tabla 1.

Criterios de Inclusión:

1). Tener el cargo de rector de una institución de educación media

2). Ejercer como rector de una institución educativa en Neiva

Criterios de Exclusión:

1). No firmar el consentimiento informado.

2). No ejercer como rector de una institución de educación media en Neiva

\section{REFERENCIAS}

Bolman, L., \& Deal, T. (1991). Leadership and management effectiveness: a multi-frame, multi-sector análisis. Human Resource Management. Recuperado de https://onlinelibrary.wiley.com/doi/10.1002/hrm.3930300406/

Bennis, W. (1989). On becoming a leader. Reading, MA: Adisson-Wesley Publishing Company Inc. http://forleadership.org/wp-content/uploads/On-Becoming-aLeader.pdf

Sánchez Manchola, I. D. (2009). Estilos de dirección y liderazgos en las organizaciones. Propuesta de uno modelo para su caracterización y análisis. Editorial Universidad del Valle. Cali, Colombia. Recuperado de: http://bibliotecadigital.univalle.edu.co/bitstream/10893/10312/3/Estilos\%20de\%20di reccion\%20y\%20liderazgo.pdf 
Tovar Trujillo, S., Vargas Paredes, S., \& González González, G. (2018). Toma de decisiones en la gestión del sector agroindustrial del bizcocho de achira en Huila (Colombia). Espacios, 4 - 15.

Wheatley, M. (1994). El liderazgo y la nueva ciencia. Granica, Barcelona. Recuperado de: www.academia.edu/download/31672790/EI_liderazgo_y_la_Nueva_Ciencia_RESUME N.doc 